\title{
Mitochondrial hexokinase and cardioprotection of the intact heart
}

\author{
C. J. Zuurbier • K. M. A. Smeele • O. Eerbeek
}

Published online: 4 April 2009

(C) The Author(s) 2009. This article is published with open access at Springerlink.com

\begin{abstract}
The interaction of hexokinase with mitochondria has emerged as a powerful mechanism in protecting many cell types against cell death. However, the role of mitochondrial hexokinase (mitoHK) in cardiac ischemia-reperfusion injury has as of yet received little attention. In this review we examine whether increased binding of hexokinase to the mitochondrion is also an integral component of cardioprotective signalling. We discuss observations in cardiac mitochondrial activation that directed us to the hypothesis of hexokinase cellular redistribution with reversible, cardioprotective ischemia, summarize the data showing that many cardioprotective interventions, such as ischemic preconditioning, insulin, morphine and volatile anesthetics, increase mitochondrial hexokinase binding within the intact heart, and discuss similarities between mitochondrial hexokinase association and ischemic preconditioning. Although most data indicate that mitochondrial hexokinase may indeed be an integral part of cardioprotection, a definitive proof for a causal relation between the amount of mitoHK and cardiac ischemia-reperfusion injury in the intact heart is eagerly awaited. When such relationship is indeed observed, the association of hexokinase with mitochondria will offer an opportunity to develop new therapies to combat ischemic cardiac diseases.
\end{abstract}

Keywords Mitochondria $\cdot$ Preconditioning $\cdot$ Heart . Hexokinase $\cdot$ Cell death

C. J. Zuurbier · K. M. A. Smeele · O. Eerbeek

Laboratory of Experimental Intensive Care \& Anesthesiology

(L.E.I.C.A.), Department of Anesthesiology and Department of

Physiology, Academic Medical Center, University of Amsterdam,

Amsterdam, The Netherlands

C. J. Zuurbier $(\bowtie)$

Department of Anaesthesiology, Academic Medical Centre,

University of Amsterdam,

Meibergdreef 9,

1105 AZ Amsterdam, The Netherlands

e-mail: c.j.zuurbier@amc.uva.nl

\section{Introduction}

In the past two decades the mitochondria have revealed their true nature in matters of life and death: not only are they the power houses providing the necessary ATP for the high-energy demand of highly organized multicellular organism, they are also the cellular dictators of cell death. Interestingly, a glycolytic enzyme, hexokinase (HK), has appeared as one of the main gatekeepers of mitochondria-induced cell death (Robey and Hay 2005). The interaction of hexokinase with mitochondria results in suppression of cell death in many cell types, and is probably one of the major mechanisms allowing unrestricted growth of advanced cancer cells which are protected against apoptosis (Pedersen 2008). Surprisingly, almost all data on the protective role of $\mathrm{HK}$ against cell death has been performed in cellular studies using primarily $\mathrm{H}_{2} \mathrm{O}_{2}$ as the oxidative injury stimulus. Little information exists as to whether mitochondrial $\mathrm{HK}$ is protective against oxidative injury under more physiological conditions. There is no information available as to whether mitoHK may actually provide survival benefit against ischemia-reperfusion (I/R) in the intact heart. Whereas such an endogenous protection mechanism offers a challenge to oncologists to combat in cancer cells, it may offer a therapeutic opportunity for cardiologists in the setting of cardiovascular ischemic disease. This minireview focuses on recent evidence that mitochondrial hexokinase may be an integral component of cardioprotective signalling in the heart.

\section{Why could there be a role for mitochondrial HK in cardioprotection?}

Ischemic preconditioning is one of the strongest interventions to bring the heart in a protected state. It entails the application of short, reversible periods of ischemia $(<15 \mathrm{~min})$ before a long, irreversible period of ischemia 
(>20 min) that results in cell death. It was discovered in 1986 by Murry and coworkers, who showed a reduction in cell death by $60-70 \%$. Many of the signals that induce preconditioning converge on the mitochondrion (Murphy and Steenbergen 2008). In addition, alterations in glycolysis were also found to be a signature of ischemic preconditioned hearts (Murry et al. 1986). We also found this interaction between changes in glycolysis and mitochondrial function with reversible ischemia that induces preconditioning. Work from our laboratory, focussing on the activation of mitochondrial oxygen consumption with instantaneous increases in workload (Van Beek et al. 1998), demonstrated that glycolysis needs to be active to observe changes in mitochondrial activation after a reversible period of ischemia, which were not observed after irreversible ischemia (Zuurbier and Van Beek 1997; Zuurbier and Ince 2002). Thus, alterations in glycolysis induced by reversible ischemia resulted in a specific change in mitochondrial activation that is characteristic of heart tissue in the cardioprotective state. What type of changes in glycolysis could possibly be associated with protection against $I / R$ injury? It has been reported that cardioprotection induced by preconditioning resulted in decreased anaerobic glycolysis and a decreased build-up of fructose-6-phosphate and lactate, despite normal or even elevated levels of glucose and glucose-6-phosphate during the sustained ischemia (Murry et al. 1986; Jennings et al. 2001; Vogt et al. 2002). Thus preconditioning induced alterations in glycolysis that are localized just after the first step of glycolysis, i.e. the phosphorylation of glucose by HK. We have shown that the decreased build-up of glycolytic intermediates after the $\mathrm{HK}$ reaction is not due to an increased channelling of these glycolytic intermediates into the pentose phosphate pathway (Zuurbier et al. 2004), indicating that the changes described above are probably the result of changes within the control of glycolysis itself.

Regulation of glycolysis by ischemic preconditioning may involve enzyme translocation, with HK being a likely candidate. Especially the translocation of $\mathrm{HK}$ to the mitochondria could explain many of the phenomena described for ischemic preconditioning. First of all, binding of $\mathrm{HK}$ to mitochondria protects against apoptosis induced by oxidative stress (Majewski et al. 2004a, b; Pastorino and Hoek 2003), which complements older literature by Pedersen et al. 2002 showing that mitochondrial hexokinase provides survival benefit in cancer. Thus, translocation of HK to the mitochondrion may constitute part of the cardioprotective phenotype of ischemic preconditioning. In addition, the association of $\mathrm{HK}$ with mitochondria is kinetically advantageous during normoxia (increased affinity for the substrate ATP, decreased sensitivity to inhibition by its product glucose-6-phopshate) and brings glycolysis actually under the control of oxidative phosphorylation
(Wilson 1995). This may partly explain (in addition to the effects of increased GLUT4 transporters in the sarcolemma) the often observed increase in aerobic glycolysis of the heart during normoxia following reversible periods of ischemia (Zuurbier and Van Wezel 2008), and the accelerated decrease in anaerobic glycolysis rate during the first period of ischemia in preconditioned hearts (Murry et al. 1986). This increased coupling of glycolysis with oxidative metabolism may result in a decreased anaerobic glycolytic proton production and consequently attenuated acidosis (Wilson 2003, Lopaschuk et al. 1993), as is usually observed in preconditioned hearts.

It thus seems that increased association of hexokinase with the mitochondria within the heart may explain many of the phenomena observed with cardioprotection, offering the arguments for a critical evaluation of the role of mitochondrial HK in cardioprotection.

\section{Cardioprotective interventions and mitochondrial bound HK}

In 2005, the first study was performed that examined whether short, reversible periods of ischemia resulting in ischemic preconditioning, was associated with HK translocation to the mitochondria within the intact heart (Zuurbier et al. 2005). It was demonstrated that hexokinase activity decreased in the cytosol, whereas it increased in the mitochondrial compartment of the ischemic preconditioned heart. In addition, two other well-known cardioprotective interventions were examined: morphine and insulin administration. Both morphine and insulin were also able to redistribute $\mathrm{HK}$ from the cytosol to the mitochondrial compartment. That HK translocation involved in cardioprotective signalling was further collaborated by a subsequent study in in vivo rat hearts (Zuurbier et al. 2008). It was shown that well-known cardioprotective anesthetics such as isoflurane and sevoflurane are able to keep hexokinase at the mitochondria during sedation, whereas other non-cardioprotective anesthetics such as ketamine resulted in increases of cytosolic $\mathrm{HK}$ as compared to conscious, non-anesthetized, animals. These data clearly demonstrated that translocation of hexokinase to the mitochondria with ischemic preconditioning is operative within the intact heart and that this may very well constitute a general end-effector of cardioprotective interventions.

Current knowledge of the cellular signalling pathways that initiate ischemic preconditioning suggests a major role for activation of so-called survival kinases such as the PI3K-Akt cascade (Tong et al. 2000) and the JAK-STAT pathway (Hattori et al. 2001). Several cellular studies by Majewski et al. (2004a, b) have demonstrated that activated Akt results in translocation of $\mathrm{HK}$ to the mitochondria. It 
has been suggested that activated Akt resulted in phosphorylation and thereby inhibiting GSK-3 $\beta$, and that this inhibition resulted in the translocation of HK to the mitochondria (Pastorino et al. 2005). Alternatively, it is also possible that activated Akt directly phosphorylates HK in the cytosol and that this facilitates HK association with mitochondria (Miyamoto et al. (2008). Thus, Akt activation may closely correspond with mitochondrial HK translocation. In addition, it has been shown that Akt activation has a biphasic behaviour with ischemic preconditioning (Hausenloy et al. 2005): Akt is activated before and after, but not during the sustained ischemic period in preconditioned hearts. Fully in line with this, we have observed similar biphasic behaviour for mitochondrial $\mathrm{HK}$ activity with ischemic preconditioning: mitochondrial HK activity was increased before and after, but not during the sustained ischemic period in ischemic preconditioned hearts as compared to control hearts (Gürel et al. 2009).

In conclusion, cardioprotective signalling in the intact heart appears to be associated with increases of hexokinase at the level of the mitochondria in a fashion similar as to that found to provide survival benefit in other cell types including malignant tumour cells, and that the HK translocation may be regulated by the RISK pathway (Fig. 1).

\section{Possible mechanisms of mitoHK-mediated cardioprotection against $\mathbf{I} / \mathrm{R}$ injury}

Hexokinase may offer protection against ischemiareperfusion injury by increases in its activity, independent of its binding to mitochondria. The increased HK activity may provide protection through improved ATP production by activating glycolysis. In an elegant study by Sun et al. (2008) it was shown that overexpression of truncated HK proteins lacking the mitochondrial binding domains could indeed partly explain the protective effects of overexpressed full length $\mathrm{HK}$ proteins against $\mathrm{H}_{2} \mathrm{O}_{2}$ treatment in HEK293 cells. Similarly, overexpressing of yeast hexokinase, which does not bind to mitochondria, protected hypoxic cardiomyocytes against contractile dysfunction (Ye et al. 2005). However, whether these protective effects of increased HK activity observed in cellular studies (where glucose is usually the sole substrate provided) translate to a more physiological model of ischemia-reperfusion in the intact heart ischemia is unclear. Liang et al. (2002) has shown that overexpressing yeast hexokinase did not protect against contractile dysfunction following hypoxia in the Langendorff-perfused mouse heart. Thus it is unclear whether an increased HK activity per se will always cause survival benefit against an ischemia-reperfusion insult in the intact heart. This is commensurate with the well-known dual role (detrimental or beneficial) of anaerobic glycolysis in cardiac ischemia-reperfusion injury (Cross et al. 1996).

$\mathrm{I} / \mathrm{R}$ injury is initiated by calcium overload and generation of reactive oxygen species, which result in activation of proteases (e.g. caspases, calpains), mitochondrial depolarization and mitochondrial permeability transition (MPT) pore opening. Although still elusive, the MPT pore is suggested to be composed of VDAC, ANT, cyclophilin D and HK (Green and Kroemer 2004). One mechanism, through which mitochondrial binding of $\mathrm{HK}$ could be protective, is by occupying MPT-VDAC binding sites which otherwise would be occupied by the pro-apoptotic
Fig. 1 Proposed view for how cardioprotective interventions through cellular signalling translocate hexokinase (HK) to the mitochondria and how increases in mitochondrial HK (mitoHK) may offer protection against ischemia-reperfusion injury. IPC ischemic preconditioning, IMM inner mitochondrial membrane, OMM outer mitochondrial membrane, CypD cyclophilin D, ANT adenine nucleotide translocator, VDAC voltage dependent anion channel, IRS insulin receptor substrate, GPCR G proteincoupled receptor, PI3K phosphoinositide 3-kinase, PKC protein kinase $\mathrm{C}$, GSKhh- $3 \beta$ glycogen synthase kinase, $\Delta \psi$ mitochondrial membrane potential, ROS reactive oxygen species

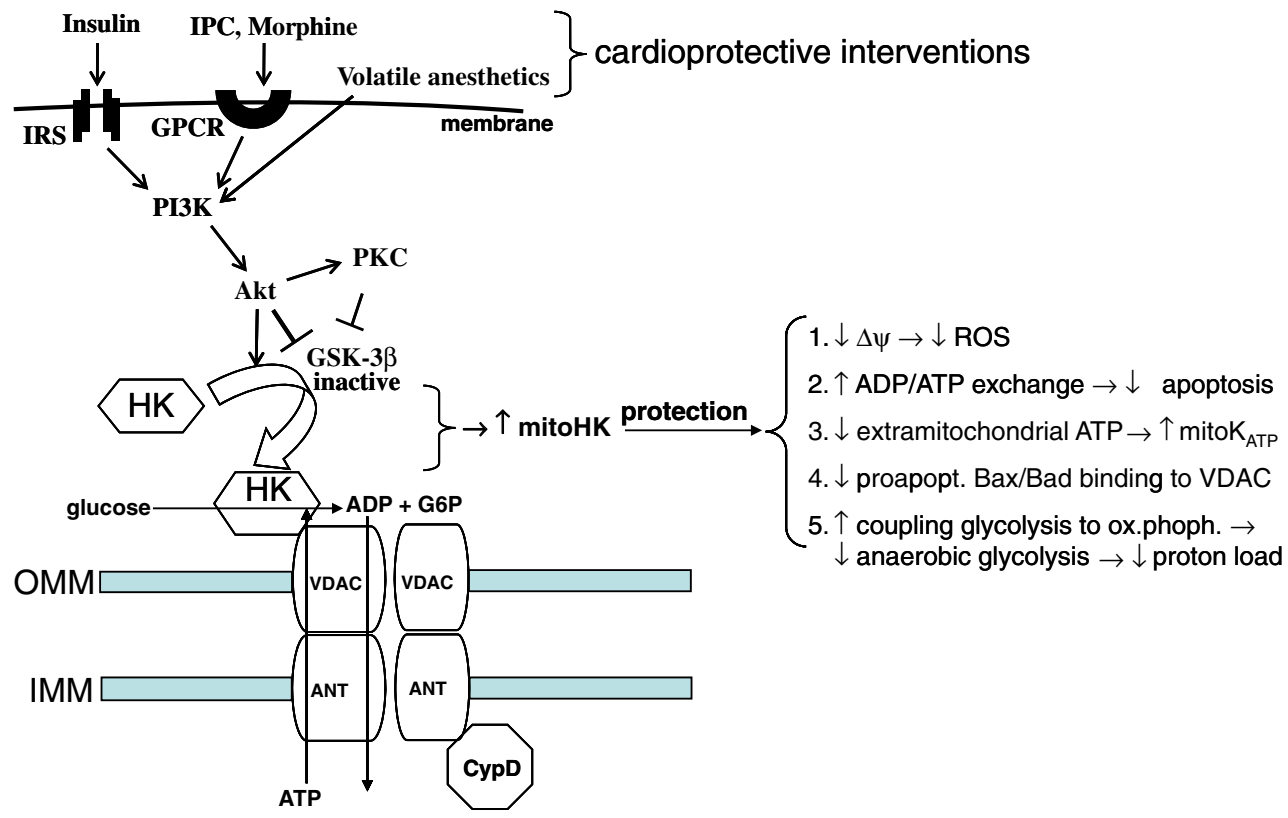


proteins Bax/Bak (Pastorino and Hoek 2008). Whether VDAC is an essential component in this scenario was recently contested by Chiari et al. (2008), showing that HK detachment from mitochondria triggers apoptosis independent of VDAC. These authors imply that mitoHK protects by affecting the configuration of the two other components of the MPT, i.e. ANT and/or cyclophylin D, although the precise mechanism was not characterized. One possibility may be that hexokinase activity drains mitochondrial ATP for the phosphorylation of glucose, thereby facilitating ATP to move out of the mitochondria and ADP to move in, and stimulating ATP/ADP exchange. It has been shown by Van der Heiden et al. (1999) that an early event in apoptosis is a defect in ATP/ADP exchange.

Alternatively, an attractive hypothesis is that mitoHK serves as a specific antioxidant against mitochondrial radical production. Da-Silva et al. (2004) have demonstrated that mitochondrial bound hexokinase reduced mitochondrial membrane potential and ROS production in brain mitochondria. Similar findings have been recently observed in heart mitochondria (Santiago et al. 2008), and the reduction of oxidative stress has been recently implicated as a likely candidate for inhibition of the MPT with ischemic preconditioning (Clarke et al. 2008). Increases of mitochondrial hexokinase functionally mimics increases in mitochondrial uncoupling, a phenomenon which is currently regarded as one of the mechanisms providing ischemic preconditioning-induced cardioprotection (Sack 2006). Finally, it has recently been reported that hearts of hypoxiatolerant fish have elevated amounts of HK bound to mitochondria. These authors proposed that the high level of mitochondrial HK serves to create microenvironments with a low ATP/ADP ratio, which could lead to the opening of mitoK $\mathrm{K}_{\mathrm{ATP}}$ channels that occur in hypoxic-tolerant fish hearts (Treberg et al. 2007). Such an opening of mitoK $\mathrm{K}_{\mathrm{ATP}}$ channels also figures as one of the main ischemic preconditioning triggering mechanism (Garlid et al. 1997).

Overall, many of the purported protective mechanisms against cell death of mitochondrial bound HK (Fig. 1) are similar to the mechanisms through which ischemic preconditioning and other cardioprotective interventions are currently proposed to operate.

\section{Conclusions}

We have reviewed here the current literature which shows that the binding of hexokinase to mitochondria is induced during many cardioprotective interventions in the intact heart. There are also many similarities between the cellular signalling which leads to the cardioprotective mechanism of ischemic preconditioning, and the cellular signalling which leads to the protective mechanisms of HK translocation and its binding to the mitochondria, thus further supporting a role of HK in cardioprotection. Further studies examining a causal relationship between the amount of mitoHK and cardiac ischemia-reperfusion injury are necessary to test the hypothesis that mitochondrial hexokinase is indeed an important player in the field of cardioprotection and cardiac ischemic diseases.

Open Access This article is distributed under the terms of the Creative Commons Attribution Noncommercial License which permits any noncommercial use, distribution, and reproduction in any medium, provided the original author(s) and source are credited.

\section{References}

Chiari F, Castellaro D, Marin O, Petronilli V, Brusilow WS, Juhaszova M, Sollott SJ, Forte M, Bernardi P, Rasola A (2008) PLoS ONE 3:e1852

Clarke SJ, Khaliulin I, Das M, Parker JE, Heesom KJ, Halestrap AP (2008) Circ Res 102:1082-1090

Cross HR, Opie LH, Radda GK, Clarke K (1996) Circ Res 78:482-491

Da-Silva WS, Gómez-Puyou A, de Gómez-Puyou MT, MorenoSanchez R, De Felice FG, de Meis L, Oliveira MF, Galina A (2004) J Biol Chem 279:39846-39855

Garlid KD, Paucek P, Yarov-Yarovoy V, Murray HN, Darbenzio RB, D'Alonzo AJ, Lodge NJ, Smith MA, Grover GJ (1997) Circ Res 81:1072-1082

Green DR, Kroemer G (2004) Science 305:626-629

Gürel E, Smeele KM, Eerbeek O, Koeman A, Demirci C, Hollmann MW, Zuurbier CJ (2009) J Appl Physiol. doi:10.1152/japplphysiol. 90537.2008

Hattori R, Maulik N, Otani H, Zhu L, Cordis G, Engelman RM, Siddiqui MA, Das DK (2001) J Mol Cell Cardiol 33:19291936

Hausenloy DJ, Tsang A, Mocanu MM, Yellon DM (2005) Am J Physiol Heart Circ Physiol 288:H971-H976

Jennings RB, Sebbag L, Schwartz LM, Crago MS, Reimer KA (2001) J Mol Cell Cardiol 33:1571-1588

Liang Q, Donthi RV, Kralik PM, Epstein PN (2002) Cardiovasc Res 53:423-430

Lopaschuk GD, Wambolt RB, Barr RL (1993) J Pharmacol Exp Ther 264:135-144

Majewski N, Nogueira V, Robey RB, Hay N (2004a) Mol Cell Biol 24:730-740

Majewski N, Nogueira V, Bhaskar P, Coy PE, Skeen JE, Gottlob K, Chandel NS, Thompson CB, Robey RB, Hay N (2004b) Mol Cell 16:819-830

Miyamoto S, Murphy AN, Brown JH (2008) Cell Death Diff 15:521-529

Murphy E, Steenbergen C (2008) Physiol Rev 88:581-609

Murry CE, Jennings RB, Reimer KA (1986) Circulation 74:11241136

Pastorino JG, Hoek JB (2003) Curr Med Chem 10:1535-1551

Pastorino JG, Hoek JB (2008) J Bioenerg Biomembr 40:171-182

Pastorino JG, Hoek JB, Shulga N (2005) Cancer Res 65:10545-10554

Pedersen PL (2008) J Bioenerg Biomembr 40:123-126

Pedersen PL, Mathupala S, Rempel A, Geschwind JF, Ko YH (2002) Biochim Biophys Acta 1555:14-20

Robey RB, Hay N (2005) Cell Cycle 4:654-658

Sack MN (2006) Cardiovasc Res 72:210-219 
Santiago APSA, Chaves EA, Oliveira MF, Galina A (2008) Biochimie 90:1566-1577

Sun L, Shukair S, Naik TJ, Moazed F, Ardehali H (2008) Mol Cell Biol 28:1007-1017

Tong H, Chen W, Steenbergen C, Murphy E (2000) Circ Res $87: 309-381$

Treberg JR, MacCormack TJ, Lewis JM, Almeida-Val VMF, Val AL, Driedzic WR (2007) Physiol Biochem Zool 80:542-550

Van Beek JHGM, Tian X, Zuurbier CJ, de Groot B, Van Echteld CJA, Eijgelshoven MHJ, Hak JB (1998) Mol Cell Biochem 184:321-344

Van der Heiden MG, Chandel NS, Schumacker PT, Thompson CB (1999) Mol Cell 3:159-167

Vogt AM, Poolman M, Ackermann C, Yildiz M, Schoels W, Fell DA, Kübler W (2002) J Biol Chem 277:24411-24419
Wilson JE (1995) Rev Physiol Biochem Pharmacol 126:65-198

Wilson JE (2003) J Exp Biol 206:2049-2057

Ye G, Donthi RV, Metreveli NS, Epstein PN (2005) Cardiovas Toxicol 5:293-300

Zuurbier CJ, Van Beek JH (1997) Circ Res 81:69-75

Zuurbier CJ, Ince C (2002) Pflügers Arch 443:908-916

Zuurbier CJ, Van Wezel HB (2008) Cardiovasc Drugs Ther 22:125-131

Zuurbier CJ, Eerbeek O, Goedhart PT, Struys EA, Verhoeven NM, Jacobs C, Ince C (2004) Cardiovasc Res 62:145-153

Zuurbier CJ, Eerbeek O, Meijer AJ (2005) Am J Physiol Heart Circ Physiol 289:H496-H499

Zuurbier CJ, Keijzers PJM, Koeman A, Van Wezel HB, Hollmann MW (2008) Anesth Analg 106:135-142 